Die FMH setzt sich für eine hohe Qualität in unserem Gesundheitswesen und einen unverfälschten Qualitätswettbewerb ein. Dies ist jedoch nur möglich, wenn Qualitätsdaten korrekt erhoben und ausgewertet werden. Eine Veröffentlichung von Qualitätsdaten hat den diesbezüglichen Empfehlungen der Schweizerischen Akademie der Medizinischen Wissenschaften SAMW zu folgen, wenn nicht in Kauf genommen werden will, dass mehr Schaden als Nutzen angerichtet wird. Im Zentrum müssen stets die Bedürfnisse von Patientinnen und Patienten stehen. Die FMH unterstützt daher eine zielgruppenspezifischeTransparenz. Nicht alle Qualitätsdaten sind für die verschiedenen Anspruchsgruppen gleich interpretierbar und nützlich. Es eignen sich somit nicht alle Qualitätsdaten für die breite Veröffentlichung. Unvollständige, inkorrekte oder inkorrekt analysierte bzw. publizierte medizinische Qualitätsdaten verzerren die Realität und verhindern, dass die Ärzteschaft und die Spitäler/Kliniken die gute Qualität ihrer Leistungen aufzeigen können. Solche Fehlanreize führen unweigerlich zu fatalen Fehlentwicklungen, was sich qualitätsmindernd auswirkt. Nicht zuletzt wird so auch die Öffentlichkeit getäuscht und ihr Vertrauen in das Gesundheitssystem in ungerechtfertigter Art und Weise geschwächt. Eine positive Wirkung durch richtige Anreize kann nur durch die Veröffentlichung von vollständigen, korrekten und für die Empfänger verständlichen Qualitätsdaten erreicht werden.

\title{
Plädoyer für faire Spital- und Klinikvergleiche
}

\section{Petra Busch}

Dr. rer. pol., Geschäftsleiterin ANO

Der Bedarf an fassbaren, prägnanten Informationen über die Leistung von Spitälern, Kliniken und Ärzteschaft steigt laufend. Diese Nachfrage ruft neue Spitalsuchund Spitalvergleichsportale auf den Plan. Sie alle berufen sich in der Schweiz auch auf die Ergebnisse der ANQ-Qualitätsmessungen; einzelne bilden daraus gar Rankings. Solche Ranglisten lehnt der ANQ jedoch entschieden ab, weil sie aus wissenschaftlicher Sicht nicht möglich sind.

Der ANQ koordiniert und führt seit 2009 landesweit einheitliche Qualitätsmessungen in der stationären Akutsomatik, Rehabilitation und Psychiatrie durch. Damit setzt er die Vorgaben des Krankenversicherungsgesetzes (KVG) um. Bei seiner Tätigkeit ist der ANQ grösstmöglicher wissenschaftlicher Sorgfalt und Transparenz verpflichtet. Entsprechend präsentiert und kommuniziert er die Messergebnisse: eingebettet in den Gesamtkontext, grafisch verständlich dargestellt, Lesehilfen inklusive.

Bei der Ergebniskommunikation sind faire Spital- und Klinikvergleiche oberstes Gebot. Der ANQ beobachtet die Entwicklung im Bereich der Spitalsuch- und Spitalvergleichsportale deshalb aufmerksam und missbilligt eine nicht autorisierte, reduzierte oder unsachgemässe Verwendung seiner Messresultate durch Dritte.
Die ANQ-Messresultate eignen sich nicht für Rankings. Daraus einfache Spital- und Klinikranglisten abzuleiten ist aus mehreren Gründen unmöglich.

\section{Wesen eines Qualitätsindikators}

Bevor eine Qualitätsmessung durchgeführt wird, gilt es, den dafür geeigneten Qualitätsindikator zu bestimmen. Qualitätsindikatoren sind Instrumente zur quantitativen Bewertung wichtiger Funktionen, die sich auf das Behandlungsergebnis bei Patientinnen und $\mathrm{Pa}-$ tienten auswirken. Sie können Struktur-, Prozess- oder Ergebnisqualität abbilden, die für das Monitoring und das Qualitätsmanagement zentral sind. So schärfen sie den Blick für potentielle Problembereiche, bewirken deren Analyse, Überprüfung, Verbesserung. Die Mess- 
werte können jeweils innerhalb oder ausserhalb eines Referenzbereichs liegen. Oder unter oder über einem Referenzwert. Bei einem Indikator handelt es sich aber um kein direktes Qualitätsmass.

\section{Aspekte der Gesamtqualität}

Ob ein Qualitätsindikator ausserhalb seines etablierten Einsatzgebiets angewandt werden kann, beispielsweise für Spitalplanung oder Pay for Performance (qualitätsabhängige Vergütung), wird national wie international wissenschaftlich kontrovers diskutiert. Fakt ist: Der alles umfassende Indikator, der klipp und klar Aufschluss über die Gesamtqualität eines Spitals oder einer Klinik geben könnte, existiert nicht - und es wird ihn nach heutigem Erkenntnisstand auch nie geben. Somit bilden also die einzelnen ANQ-Messresultate immer nur spezifische Qualitätsaspekte eines Spitals oder einer Klinik im Kontext der jeweiligen Messung ab. Die Ergebnisse auf die Gesamtqualität einer Institution hochzurechnen oder mit einer entsprechenden Publikation bei Internetusern dieses Bild zu erwecken ist unzulässig.

\section{Chancen der ANQ-Indikatoren}

Die Indikatoren, welche vom ANQ gewählt wurden, eignen sich primär für die Qualitätsentwicklung in Schweizer Spitälern und Kliniken: Sie liefern die Grundlagen, damit in den Institutionen gezielte Verbesserungsmassnahmen angestossen und umgesetzt werden können. Geeignet sind sie für landesweit einheitliche Messungen sowie für eine transparente Information und Ergebnispublikation, sofern die Datenqualität dafür ausreicht. Sie ermöglichen eine faire Vergleichbarkeit der Ergebnisse zwischen den einzelnen Institutionen und dem Gesamtergebnis aller Spitäler und Kliniken. Kantonen und Versicherern dienen sie als Anhaltspunkte für den Dialog mit den Institutionen.

\section{Grenzen der ANQ-Indikatoren}

Die Ergebnisse der ANQ-Messungen können weder für Ressourcenallokation noch für Pay for Performance beigezogen werden. Dafür müssten andere Indikatoren bestimmt werden. Der ANQ rät auch dringend davon ab, sie als Begründung für Sanktionen gegenüber Spitälern oder Kliniken mit schlechteren Messergebnissen als der Durchschnitt zweckzuentfremden. Denn je nach ANQ-Fachbereich, gewähltem Qualitätsindikator und dem damit verbundenen Messinstrument ist die Aussagekraft der einzelnen Messungen limitiert. Bei jeder Messung bestehen unterschiedliche Chancen und Grenzen, insbesondere Risiken von Fehlinterpretationen, wie das nachfolgende Beispiel zeigt.

\section{Beispiel: Wundinfektionsraten nach Operationen}

Swissnoso, die Vereinigung von führenden Fachleuten auf dem Gebiet der Infektionskrankheiten und der Spitalhygiene, überwacht im Auftrag des ANQ seit acht Jahren die Entwicklung von Wundinfektionen mit dem Messinstrument SSI Surveillance. Spitalinfektionen, insbesondere postoperative Wundinfektionen, werden generell mit der Leistungsqualität von Spitälern und Kliniken verbunden. Deshalb ist die transparente Veröffentlichung der Infektionsraten nach chirurgischen Eingriffen für die Bevölkerung und die Medien von grossem Interesse.

Bei Ergebnisinterpretation und Kommunikation sind gleich mehrere Faktoren zu berücksichtigen und auszuschliessen, die ein falsches Bild vermitteln können:

- Wundinfektionen müssen immer im Kontext eines chirurgischen Eingriffs betrachtet werden. Das Ziel einer Operation kann trotz eines Infekts erreicht werden - zum Beispiel Schmerzentlastung oder verbesserte Gelenkfunktionalität. Wundinfektionen nach Operationen können sowieso nie ganz vermieden werden. Einige Eingriffe sind allein schon aufgrund des Operationsgebiets risikoreicher, allen voran solche in der Dickdarm- oder Enddarmchirurgie.

- Das statistische Verfahren der Risikobereinigung ${ }^{1}$ ist nicht perfekt und kann nicht alle Unterschiede zwischen den Institutionen korrigieren.

- Die Qualität der Datenerhebungsprozesse wird mit Hilfe von standardisierten Instrumenten ermittelt (Validierung). Bei Spitälern und Kliniken mit tiefer Erfassungsqualität können die Infektionsraten verzerrt sein und müssen deshalb vorsichtig interpretiert werden.

- Die Schweizer Infektionsraten sind nur bedingt mit denjenigen aus Überwachungssystemen anderer Ländern vergleichbar. Massgebliche Unterschiede bestehen bei Methode und Qualität der Erfassung sowie bei der Überwachung nach Spitalaustritt. Weil die Schweiz meist gründlicher erfasst und länger überwacht, ergeben sich in der Schweiz teilweise höhere Raten als in anderen Ländern.

Im Argumentarium "Chancen und Grenzen der ANQ-Messun gen» zeigt der ANQ diese Thematik pro Messung/lndikator auf: www.anq.ch/messergebnisse 


\section{Positive Wirkung}

Dank den ANQ-Qualitätsmessungen erhalten Spitäler und Kliniken differenzierte Informationen, können sich mit anderen vergleichen. Die ANQ-Messungen sind für sie umsetzbar, bringen einen praktischen Nutzen im klinischen Alltag. Oft lösen die Messergebnisse Verbesserungsprozesse aus und liefern Argumentationshilfen für entsprechende Investitionen. Dazu zählen gezielte Prozessanalysen, Schulungen, die (Weiter)entwicklung von Konzepten etc. Die transparente Veröffentlichung wirkt spital- und klinikintern auf mehreren Ebenen: Sie unterstützt das Agenda-Setting, sensibilisiert, zeigt Handlungsbedarf auf, kann gar eine Kulturveränderung bewirken.

\section{Dialog statt Pranger}

Der ANQ begrüsst es, wenn die Resultate seiner Qualitätsmessungen öffentlich und sachlich diskutiert werden. Ein Diskurs über die Qualität im Schweizer Gesundheitswesen ist unumgänglich, und der ANQ leistet hier einen wichtigen Beitrag.

Dass ein wachsendes Bedürfnis nach verständlicher Qualitätsinformation besteht, kann der ANQ nachvollziehen. Simple Rangreihen dienen der sorgfältigen Qualitätsentwicklung aus vorgenannten Gründen jedoch nicht und wirken kontraproduktiv. Der ANQ
Der Nationale Verein für Qualitätsentwicklung in Spitälern und Kliniken (ANQ)

Der Nationale Verein für Qualitätsentwicklung in Spitälern und Kliniken (ANQ) koordiniert und realisiert Qualitätsmessungen in der Akutsomatik, der Rehabilitation und der Psychiatrie. Die Resultate ermöglichen eine transparente und nationale Vergleichbarkeit. Aufgrund dieser Erkenntnisse können Spitäler und Kliniken gezielt Massnahmen zur Verbesserung ihrer Qualität entwickeln. ANQ-Mitglieder sind der Spitalverband $\mathrm{H}+$, santésuisse, die Eidgenössischen Sozialversicherer, die Kantone und die Schweizerische Gesundheitsdirektorenkonferenz. Der Verein arbeitet nicht gewinnorientiert.

www.anq.ch

stellt deshalb keine einzelnen Spitäler heraus - weder im positiven noch im negativen Sinne. Spitäler mit schlechteren Werten sind allein schon durch die transparente Veröffentlichung zum Handeln aufgefordert. Dank den einheitlichen, vergleichbaren Daten aus den ANQ-Messungen haben sie die Möglichkeit, den Finger auf den wunden Punkt zu legen und sich an den Besten ihres Spital- oder Kliniktyps zu orientieren. 